\title{
A unified evaluation of iterative projection algorithms for phase retrieval
}

\author{
S. Marchesini ${ }^{1,2, \text { * }}$ \\ ${ }^{1}$ Lawrence Livermore National Laboratory, 7000 East Ave., Livermore, CA 94550-9234, USA \\ ${ }^{2}$ Center for Biophotonics Science and Technology, University of California, \\ Davis, 2700 Stockton Blvd., Ste 1400, Sacramento, CA 95817, USA
}

(Dated: October 29, 2018)

\begin{abstract}
Iterative projection algorithms are successfully being used as a substitute of lenses to recombine, numerically rather than optically, light scattered by illuminated objects. Images obtained computationally allow aberration-free diffraction-limited imaging and the possibility of using radiation for which no lenses exist. The challenge of this imaging technique is transferred from the lenses to the algorithms. We evaluate these new computational "instruments" developed for the phase-retrieval problem, and discuss acceleration strategies.
\end{abstract}

Crystallographers routinely image molecular structures of several thousand atoms by phasing the diffraction pattern of a structure replicated in a periodic system. Likewise, computationally retrieving the phase of a diffraction pattern is becoming increasingly successful at imaging - with several millions of resolution elementsobjects as complex as biological cells, nanotubes and nanoscale aerogel structures. Diffraction microscopy (the imaging of isolated objects by diffraction and computational phase retrieval) promises a 3D resolution limited only by radiation damage, wavelength, the collected solid angle and the number of x-rays or electrons collected. This capability provides an extremely valuable tool for understanding nanoscience and cellular biology. Recent estimates [1] of the dose and flux requirements of x-ray diffraction on single objects indicate that attractive resolution values (about $10 \mathrm{~nm}$ for life science and $2-4 \mathrm{~nm}$ for material science) should be possible at a modern synchrotron. Atomic resolution could be accomplished using pulses of $\mathrm{x}$-rays that are shorter than the damage process itself [2, 3] using femtosecond pulses from an x-ray freeelectron laser [4]. Alternatively the radiation damage limit could be eliminated by continuously replacing the exposed samples, such as laser-aligned molecules [5] with identical ones.

In the fields of electron microscopy [6] and astronomical imaging 7], iterative projection algorithms have been used to recover the phase information in a variety of problems. The evaluation of the aberrations in the Hubble space telescope described by Fienup in [8] remains perhaps the most prominent example of successful phase reconstructions in the astronomical community. Nugent and collaborators applied similar techniques to characterize x-ray lenses [9]. In electron diffraction microscopy [6, 10], Zuo and coworkers imaged a single isolated nanotube at atomic resolution [11], Wu et al. imaged defects at atomic resolution [12].

\footnotetext{
* Correspondence and requests for materials should be addressed to S. Marchesini: smarchesini@lbl.gov

${ }^{\dagger}$ Current address: Lawrence Berkeley National Laboratory, $1 \mathrm{Cy}-$ clotron Rd, Berkeley CA 94720, USA.
}

An important review, which attempted to integrate the approaches of the optical and crystallographic communities, appeared in 1990 [13]. The connection was made between the "solvent-flattening" or "density-modification" techniques of crystallography [14] and the compact support requirements of the iterative projection algorithms. The importance of fine sampling of the intensity of the measured diffraction pattern was recognized at an early stage [15].

The observation by Sayre in 1952 [16] that Bragg diffraction undersamples the diffracted intensity pattern was important and led to more specific proposals by the same author for x-ray diffractive microscopy of nonperiodic objects [17, 18]. These ideas, combined with the rapid development of computational phase retrieval in the wider optics community, especially the "support constraint" [6, 7, 19, 20], enabled the first successful use of coherent x-ray diffraction microscopy (CXDM).

Since the first proof of principle demonstration of CXDM by a team at Stony Brook 21], a number of groups have been working to bring these possibilities into reality.

Robinson and co-workers at the University of Illinois have applied the principles of CXDM to hard x-ray experiments on microcrystalline particles. Such data have been reconstructed tomographically to produce a 3D image at 80 and more recently $40 \mathrm{~nm}$ resolution 22, 23]. Miao (now at UCLA) and co-workers made considerable progress in pushing the CXDM method at Spring- 8 Japan to higher resolution in $2 \mathrm{D}(7 \mathrm{~nm})$, higher x-ray energies, and to a limited form of 3D [24]. They have also made the first application of CXDM to a biological sample [25].

A diffraction chamber dedicated to diffraction microscopy [26] has been used to image biological cells [27, 28] at the Advanced Light Source in Berkeley [29]. Using the same chamber, a collaboration between Berkeley and Livermore labs and Arizona State University produced 3D imaging at $10 \times 10 \times 40 \mathrm{~nm}$ resolution of test samples [30] as well as aerogel foams [31].

In this article the computational instruments that enabled these and other results are reviewed. Section II introduces the phase problem and the experimental 
requirements for diffraction microscopy, Section [II describes the concepts of sets of images and their projectors. In Section III the iterative projection algorithms published in the literature are summarized and tested on simple geometric sets. In Section IV the connection between projection- and gradient- based methods and related acceleration strategies are discussed.

\section{THE PHASE PROBLEM}

When we record the diffraction pattern intensity scattered by an object, the phase information is missing. Apart from normalization factors, an object of density $\rho(\boldsymbol{r}), \boldsymbol{r}$ being the coordinates in the object (or real) space, generates a diffraction pattern equal to the modulus square of the Fourier transform (FT) $\tilde{\rho}(\boldsymbol{k})$ :

$$
\begin{aligned}
& I(\boldsymbol{k})=|\tilde{\rho}(\boldsymbol{k})|^{2} \\
& I(\boldsymbol{k})=\tilde{\rho}^{\dagger}(\boldsymbol{k}) \tilde{\rho}(\boldsymbol{k}),
\end{aligned}
$$

where $\boldsymbol{k}$ represent the coordinate in the Fourier (or Reciprocal) space. The inverse Fourier transform (IFT) of the measured intensity $I$ provides the autocorrelation $\rho(-\boldsymbol{r}) * \rho(\boldsymbol{r})$ of the object:

$$
\operatorname{IFT}[I(\boldsymbol{k})]=\rho(-\boldsymbol{r}) * \rho(\boldsymbol{r}) .
$$

The phase-retrieval problem consists of solving $\tilde{\rho}$ in Eq. (1) or $\rho$ in Eq. (2), using some extra prior knowledge. In diffraction microscopy, solving such problem is performed with giga-element large-scale optimization algorithms, described in the following section.

Since the intensity represents the FT of the autocorrelation function, and the autocorrelation is twice as large as the object, the diffraction pattern intensity should be sampled at least twice as finely as the amplitude to capture all possible information on the object. Finer sampling adds a 0-padding region around the recovered autocorrelation function, which adds no further information (Shannon theorem). Less than critical sampling in the Fourier domain causes aliasing in the object space. A periodic repetition of the same structure provides a stronger signal, enabling the measurement of the diffraction pattern before the structure is damaged. However, while an isolated object generates a continuous diffraction pattern that can be sampled as finely as desired, a periodic repetition of the same object generates only a subset of the possible diffraction intensities. Crystallography therefore has to deal with an aliased autocorrelation function, also known as the Patterson function. This reduced information can be compensated by other prior knowledge, such as the atomic nature of the object being imaged, knowledge of a portion of the object, presence of heavy atoms, and information obtained with anomalous diffraction. Other information includes the presence of a solvent in the crystal. By varying the sampling rate of a diffraction pattern it was shown [18, 32, 33] that less than critical sampling was sufficient to solve the phase problem. This was possible because the number of equations (measured intensities in Eq. (10) in the two- and threedimensional phase-retrieval problems is larger than the number of unknowns (resolution elements in the object). The number of unknowns defines the number of independent equations, or the minimum required sampling rate. Although no general proof has been provided that limited sampling removes only redundant equations, such a minimum required sampling rate suggests that when the solvent exceeds $50 \%$ of the crystal volume, the algorithms developed in the optical community, using techniques to dynamically refine the solvent regions [34] may be able obtain ab-initio structural information from crystals.

Coherence is required to properly sample the FT of the autocorrelation of the object [35]. According to the Schell theorem [36], the autocorrelation of the illuminated object obtained from the recorded intensity is multiplied by the complex degree of coherence. The beam needs to fully illuminate the isolated object, and the degree of coherence must be larger than its autocorrelation.

Diffraction microscopy solves the phase problem by using the knowledge that the object being imaged is isolated; it is assumed to be 0 outside a region called support $S$ :

$$
\rho(\boldsymbol{r})=0 \text {, if } \boldsymbol{r} \notin S .
$$

This support is equivalent to the solvent in crystallography. Equations (11) and (3) can be combined to obtain a multidimensional system of quadratic equations in the $\rho(\boldsymbol{r})$ variables:

$$
\left|\sum_{\boldsymbol{r} \in S} \rho(\boldsymbol{r}) \exp (i \boldsymbol{k} \cdot \boldsymbol{r})\right|^{2}=I(\boldsymbol{k}),
$$

which is a quadratic equation in the $\rho(\boldsymbol{r})$ variables with coefficients $c_{\boldsymbol{r}, \boldsymbol{r}^{\prime}}(\boldsymbol{k})=\cos \left(\boldsymbol{k} \cdot\left(\boldsymbol{r}-\boldsymbol{r}^{\prime}\right)\right)$ :

$$
\sum_{\boldsymbol{r}, \boldsymbol{r}^{\prime} \in S} c_{\boldsymbol{r}, \boldsymbol{r}^{\prime}}(\boldsymbol{k}) \rho(\boldsymbol{r}) \rho^{*}\left(\boldsymbol{r}^{\prime}\right)=I(\boldsymbol{k}) .
$$

Each value of $I(\boldsymbol{k})$ in reciprocal space defines an ellipsoid (Eq. (5)) in the multidimensional space of the unknowns $\rho(\boldsymbol{r}),\{\boldsymbol{r} \in S\}$. If the number of independent equations equals the number of unknowns, the system has a single solution $\rho(\boldsymbol{r})$. The intersection of these ellipsoids forms our solution. Unfortunately this system of equations is difficult to solve, and has an enormous number of local minima. Constant phase factors, inversion with respect to the origin (enantiomorphs), and origin shifts $\rho\left( \pm \boldsymbol{r}+\boldsymbol{r}_{\mathbf{0}}\right) e^{i \phi_{0}}$ are undetermined and considered equivalent solutions. The presence of multiple non-equivalent solutions in two- and higher- dimensional phase retrieval problems is rare [37]; it occurs when the density distribution of the object can be described as the convolution of two or more non-centrosymmetric distributions. Simple homometric structures for which the phase problem is 
not unique [38] exist in nature, but such non-uniqueness is less likely for more complex structures.

The presence of noise and limited prior knowledge (loose constraints) increases the number of solutions within the noise level and constraints. Confidence that the recovered image is the correct and unique one can be obtained by repeating the phase-retrieval process using several random starts. Repeatability of the recovered images as a function of resolution measures the effective phase-retrieval transfer function [27, 30], which can be decomposed in unconstrained amplitudes modes [27] and phase aberrations [39].

In the early 1980s, the development of iterative algorithms with feedback by Fienup, produced a remarkably successful optimization method capable of extracting phase information [7, 20, 40]. The important theoretical insight that these iterations may be viewed as projections in Hilbert space [41, 42] has allowed theoreticians to analyse and improve on the basic Fienup algorithm [43, 44, 45, 46].

These algorithms try to find the intersection between two sets, typically the set of all the possible objects with a given diffraction pattern (modulus set), and the set of all the objects that are constrained within a given area or support volume (or outside a solvent region in crystallography). The search for the intersection is based on the information obtained by projecting the current estimate on the two sets. An error metric is obtained by evaluating the distance between the current estimate and a given set. The error metric and its gradient are used in conjugate-gradient (CG) -based methods such as SPEDEN [4].

\section{SETS, PROJECTORS AND METRICS}

An image of a density distribution can be described as a sequence of $n$ pixel values. For an image of $n$ pixels, there are $n$ coordinates. The magnitude of the density at a pixel defines the value of that coordinate. Thus a single vector in this $n$-dimensional space defines an image. For complex images the number of coordinates increases by a factor of 2 . Axes of the multidimensional space are formed by any sequence of $n$-pixels with all but one pixel equal to 0 . An example is $\boldsymbol{x}=(x, 0,0)$ in 3 -pixel solution space. The origin of this space is the image with all the pixels equal to 0 . The components on these axes form the real or object space. The same object can be described in terms of any another $n$-dimensional orthogonal (or linearly independent) bases. Axes can be rotated, shifted, inverted and so on, and the proper linear transform must be applied to obtain the components in the new basis. The basis used to describe the image must have at least $n$ components, but more can be used if it helps to describe the properties of the algorithm. For example the values could be left to have a real and an imaginary component, doubling the number of dimensions used to describe the object.

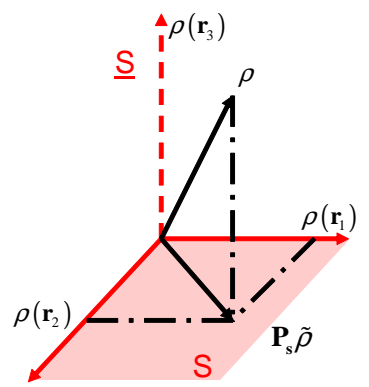

(a)

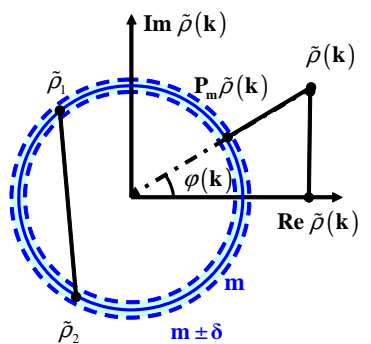

(b)
FIG. 1: Examples of sets and projectors: (a) Support: The axes represent the values on 3 pixels of an image $\rho$ known to be 0 outside the support $S$. The vertical axis $\rho\left(\boldsymbol{r}_{3}\right)$ represents a pixel outside $\left(\boldsymbol{r}_{3} \in \underline{S}\right)$, while the horizontal plane represents pixels inside $S$. The projection on this set is performed simply by setting to 0 all the pixels outside the support. (b) Modulus: A pixel (in Fourier space) with a given complex value is projected on the closest point on the circle defined by the radius $m$. If there is some uncertainty in the value of the radius $m \pm \delta$, the circle becomes a band. The circle is a nonconvex set, since the linear combination between two points on the same set $\rho_{1}$ and $\rho_{2}$ does not lie on the set. Also represented in the figure is the projection on the real axis (reality projection).

One important basis is the momentum or Fourier space. While the vector in the $n$-dimensional space representing an image is unaltered on transforming from real to reciprocal space, its components in the new axes are altered (Fourier-transformed). The distance between two points in the $n$-dimensional space is independent of this transformation (Parseval theorem). The lengths and the angles between vectors will be our guide to describe the behavior, convergence and error metrics of these algorithms.

We consider two sets, $S$ (support) and $M$ (modulus). When the image belongs to both sets simultaneously, we have reached a solution. If the properties of the object being imaged are known a-priori to be limited in a support region, we know that in the $n$-dimensional space of the pixel values, some values must be zero. Images that satisfy this rule (Eq. (3)) form the support constraint set. A projection onto this set $\left(\boldsymbol{P}_{s}\right)$ involves setting to 0 the components outside the support, while leaving the rest of the values unchanged (Fig. 1(a)):

$$
\boldsymbol{P}_{s} \rho(\boldsymbol{r})= \begin{cases}\rho(\boldsymbol{r}) & \text { if } \boldsymbol{r} \in S \\ 0 & \text { otherwise, }\end{cases}
$$

and its complementary projector $\boldsymbol{P}_{\underline{s}}=\boldsymbol{I}-\boldsymbol{P}_{s}$.

The values in every pixel in Fourier space can be described using two components, the real and imaginary parts, or amplitude and phase, both defining a point in a complex plane. In an intensity measurement we obtain the amplitude or modulus in every pixel that defines a circle in a complex plane. These circles define the modulus 


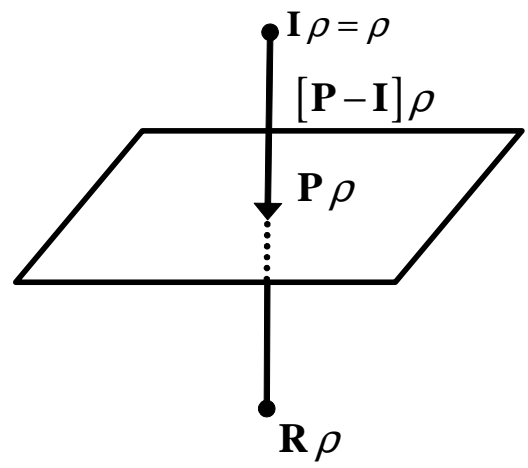

FIG. 2: The reflector applies the same step as the projector $(\boldsymbol{P}-\boldsymbol{I})$ twice: $\boldsymbol{R} \rho=\boldsymbol{I} \rho+2[\boldsymbol{P}-\boldsymbol{I}] \rho$

constraint (Fig. 1(b)). When every complex-valued pixel lies on the circle defined by the corresponding modulus, the image satisfies this constraint and it belongs to the modulus set. Segments joining two points on a circle do not belong to the circle; therefore the linear combination of two images is outside the set: the set is non-convex. These sets are problematic because of the presence of local minima and undefined projections.

The projection of a point in each complex plane onto the corresponding circle is accomplished by taking the point on the circle closest to the current one, setting the modulus to the measured one $\sqrt{I(\boldsymbol{k})}$, and leaving the phase unchanged (Fig. 1(b)):

$$
\tilde{\boldsymbol{P}}_{m} \tilde{\rho}(\boldsymbol{k})=\tilde{\boldsymbol{P}}_{m}|\tilde{\rho}(\boldsymbol{k})| e^{i \varphi(\boldsymbol{k})}=\sqrt{I}(\boldsymbol{k}) e^{i \varphi(\boldsymbol{k})},
$$

where we have defined the reciprocal space representation of the projector:

$$
\boldsymbol{P}_{m}=\mathcal{F}^{-1} \tilde{\boldsymbol{P}}_{m} \mathcal{F}
$$

and $\mathcal{F}$ and $\mathcal{F}^{-1}$ represent the forward and inverse Fourier transforms respectively.

This operator is demonstrated to be a projector on the non-convex (Fig. 1(b) set of the magnitude constraint [48]. The same paper discusses the problems of multi valued projections for non-convex sets, which do not satisfy the requirements for gradient-based minimization algorithms, and the related non-smoothness of the squared set distance metric, which may lead to numerical instabilities. See also [49] for a follow-up discussion on the non-smooth analysis.

A projector $\boldsymbol{P}$ is an operator that takes to the closest point of a set from the current point $\rho$. A repetition of the same projection is equal to one projection alone $\left(\boldsymbol{P}^{\mathbf{2}}=\boldsymbol{P}\right)$; its eigenvalues must therefore be $\lambda=0,1$. Another operator used here is the reflector $\boldsymbol{R}=\boldsymbol{I}+2[\boldsymbol{P}-\boldsymbol{I}]=2 \boldsymbol{P}-\boldsymbol{I}$, which applies the same step as the projector but moves twice as far (Fig 2). In the case of the support constraint, the whole image space can be described in terms of the eigenvectors of the corresponding linear projector. These eigenvectors with eigenvalues of $1(0)$ are the images with all the pixels equal to 0 , except for one pixel inside (outside) the support. The modulus projector is a non-linear operator:

$$
\begin{aligned}
P_{m}(a+b) & \neq P_{m}(a)+P_{m}(b) \\
P_{m}(\alpha a) & \neq \alpha P_{m}(a),
\end{aligned}
$$

and it cannot be described in terms of eigenvalues and eigenvectors.

The Euclidean length $\|\rho\|$ of a vector $\rho$ is defined as:

$$
\|\rho\|^{2}=\rho^{\dagger} \cdot \rho=\sum_{\boldsymbol{r}}|\rho(r)|^{2}=\sum_{\boldsymbol{k}}|\tilde{\rho}(k)|^{2} .
$$

The sum is extended to the measured portion of the diffraction pattern. If part of the reciprocal space is not measured, it should not be included in the sum. In fact the sum should be weighted with the experimental noise $\sigma(\boldsymbol{k})$ :

$$
\|\rho\|^{2}=\frac{\sum_{\boldsymbol{k}} \frac{1}{\sigma^{2}(\boldsymbol{k})}|\tilde{\rho}(k)|^{2}}{\sum_{\boldsymbol{k}} \frac{1}{\sigma^{2}(\boldsymbol{k})}},
$$

with $\sigma(\boldsymbol{k})=\infty$ for values of $\boldsymbol{k}$ not measured. The distance from the current point to the set $\|\boldsymbol{P} \rho-\rho\|$ is the basis for our error metric. Typically the errors in real $\left(\varepsilon_{s}\right)$ and reciprocal space $\left(\varepsilon_{m}\right)$ are defined in terms of their distance to the corresponding sets:

$$
\begin{aligned}
\varepsilon_{s}(\rho) & =\left\|\boldsymbol{P}_{s} \rho-\rho\right\|, \\
\varepsilon_{m}(\rho) & =\left\|\boldsymbol{P}_{m} \rho-\rho\right\|,
\end{aligned}
$$

or their normalized version $\bar{\varepsilon}_{x}(\rho)=\frac{\varepsilon_{x}(\rho)}{\left\|\boldsymbol{P}_{x} \rho\right\|}$. Another error metric used in the literature is given by the distance between the two sets: $\varepsilon_{s, m}(\rho)=\left\|\boldsymbol{P}_{m} \rho-\boldsymbol{P}_{s} \rho\right\|$. The projector $\boldsymbol{P}_{m}$ moves $\rho$ to the closest minimum of $\varepsilon_{m}^{2}\left(\boldsymbol{P}_{m} \rho\right)=0$, providing a simple relation with the gradient $\nabla_{\rho} \varepsilon_{m}^{2}(\rho)$ [20, 48]:

$$
\boldsymbol{P}_{\boldsymbol{m}} \rho=\rho+\left[\boldsymbol{P}_{\boldsymbol{m}}-\boldsymbol{I}\right] \rho=\rho-\frac{1}{2} \nabla_{\rho} \varepsilon_{m}^{2}(\rho),
$$

where $\nabla_{\rho} \varepsilon_{m}^{2}(\rho)$ is proportional to $\nabla_{\rho} \varepsilon_{m}(\rho)$ :

$$
\nabla_{\rho} \varepsilon_{m}^{2}(\rho)=2 \varepsilon_{m}(\rho) \nabla_{\rho} \varepsilon_{m}(\rho) .
$$

For $\tilde{\rho}(\boldsymbol{k})=0, \varepsilon_{m}$ is non differentiable, and the projector $\boldsymbol{P}_{m}$ is multivalued [48]. The presence of complex zeros $(\rho(\boldsymbol{k})=0)$ is considered of fundamental importance in the phase-retrieval problem [50], and the phase vortices associated with these zeros cause stagnation in iterative algorithms [51]. Several methods have been proposed to solve this problem [24, 39, 51, 52, 53]. Similarly the projector $\boldsymbol{P}_{s}$ minimizes the error $\varepsilon_{s}^{2}$ :

$$
\left[\boldsymbol{I}-\boldsymbol{P}_{s}\right] \rho=\rho_{\underline{s}}=\frac{1}{2} \nabla_{\rho} \varepsilon_{s}^{2}(\rho)
$$

\section{ITERATIVE PROJECTION ALGORITHMS}

Several algorithms based on these concepts have now been proposed and a visual representation of their behavior is useful to characterize the algorithm in various 
TABLE I: Summary of various algorithms

\begin{tabular}{|l|l|}
\hline \hline Algorithm & Iteration $\rho^{(n+1)}=$ \\
\hline \hline ER & $\boldsymbol{P}_{\boldsymbol{s}} \boldsymbol{P}_{\boldsymbol{m}} \rho^{(n)}$ \\
\hline SF & $\boldsymbol{R}_{\boldsymbol{s}} \boldsymbol{P}_{\boldsymbol{m}} \rho^{(n)}$ \\
\hline HIO & $\left\{\begin{array}{l}\left(\boldsymbol{I} \rho^{(n)}(\boldsymbol{r})\right. \\
\left(\boldsymbol{I} \boldsymbol{P}_{\boldsymbol{m}}\right) \rho^{(n)}(\boldsymbol{r}) \quad \boldsymbol{r} \notin S\end{array}\right.$ \\
\hline DM & $\left\{\boldsymbol{I}+\beta \boldsymbol{P}_{\boldsymbol{s}}\left[\left(1+\gamma_{s}\right) \boldsymbol{P}_{\boldsymbol{m}}-\gamma_{s} \boldsymbol{I}\right]\right.$ \\
& $\left.\quad-\beta \boldsymbol{P}_{\boldsymbol{m}}\left[\left(1+\gamma_{m}\right) \boldsymbol{P}_{\boldsymbol{s}}-\gamma_{m} \boldsymbol{I}\right]\right\} \rho^{(n)}$ \\
\hline ASR & $\frac{1}{2}\left[\boldsymbol{R}_{\boldsymbol{s}} \boldsymbol{R}_{\boldsymbol{m}}+\boldsymbol{I}\right] \rho^{(n)}$ \\
\hline HPR & $\frac{1}{2}\left[\boldsymbol{R}_{\boldsymbol{s}}\left(\boldsymbol{R}_{\boldsymbol{m}}+(\beta-1) \boldsymbol{P}_{\boldsymbol{m}}\right)\right.$ \\
& $\left.+\boldsymbol{I}+(1-\beta) \boldsymbol{P}_{\boldsymbol{m}}\right] \rho^{(n)}$ \\
\hline RAAR & {$\left[\frac{1}{2} \beta\left(\boldsymbol{R}_{\boldsymbol{s}} \boldsymbol{R}_{\boldsymbol{m}}+\boldsymbol{I}\right)+(1-\beta) \boldsymbol{P}_{\boldsymbol{m}}\right] \rho^{(n)}$} \\
\hline \hline
\end{tabular}

situations, in order to help choose the most appropriate one for a particular problem. In this section the projection algorithms published in the literature are summarized (see also Table I) and tested on simple geometrical sets.

The following algorithms require a starting point $\rho^{0}$, which is generated by assigning a random phase to the measured object amplitude (modulus) in the Fourier domain $|\tilde{\rho}(\boldsymbol{k})|=m(\boldsymbol{k})=\sqrt{I(\boldsymbol{k})}$. The first algorithm called error reduction (ER) (Gerchberg and Saxton [6, 41, 54]) is simply (Fig. 3(a)):

$$
\rho^{(n+1)}=\boldsymbol{P}_{\boldsymbol{s}} \boldsymbol{P}_{\boldsymbol{m}} \rho^{(n)}
$$

and by projecting back and forth between two sets, it converges to the local minimum. The name of the algorithm is due to the steps moving along the gradient of the error metric (see Eq. (13) ):

$$
\boldsymbol{P}_{s} \boldsymbol{P}_{m} \rho=\boldsymbol{P}_{s} \rho-\frac{1}{2} \nabla_{s} \varepsilon_{m}^{2}(\rho),
$$

where $\nabla_{s}=\boldsymbol{P}_{s} \nabla$ is the component of the gradient in the support. Figure 3(a) shows that the step size is far from optimum, but that it guarantees linear convergence. A line search along this gradient direction would considerably speed up the convergence to a local minimum and will be discussed in Section IV.

The solvent flipping (SF) algorithm 55] is obtained by replacing the support projector $\boldsymbol{P}_{s}$ with its reflector $\boldsymbol{R}_{s}=2 \boldsymbol{P}_{s}-\boldsymbol{I}$ (Fig. 3(b) :

$$
\rho^{(n+1)}=\boldsymbol{R}_{\boldsymbol{s}} \boldsymbol{P}_{\boldsymbol{m}} \rho^{(n)},
$$

which multiplies the charge density $\rho$ outside the support by -1 . The hybrid input-output (HIO) [7, 20] (Fig. [3(c) is based on non-linear feedback control theory and can be expressed as:

$$
\rho^{(n+1)}(x)= \begin{cases}\boldsymbol{P}_{\boldsymbol{m}} \rho^{(n)}(x) & \text { if } x \in S, \\ \left(\boldsymbol{I}-\beta \boldsymbol{P}_{\boldsymbol{m}}\right) \rho^{(n)}(x) & \text { otherwise. }\end{cases}
$$

Equations (13) and (15) can be used to describe the steps $\left(\Delta \rho=\rho^{(n+1)}-\rho^{(n)}\right)$ in terms of the gradients of the error metrics. In Section IV it will be shown that this algorithm seeks the saddle point:

$$
\min _{\rho_{s}} \max _{\rho_{\underline{s}}} \mathcal{L}(\rho), \quad \mathcal{L}(\rho)=\varepsilon_{m}^{2}(\rho)-\varepsilon_{s}^{2}(\rho)
$$

by moving in the descent-ascent direction $\left(\left[-\boldsymbol{P}_{s}+\right.\right.$ $\left.\beta \boldsymbol{P}_{\underline{s}}\right] \nabla \mathcal{L}$ ) (see Section $[\mathrm{IV}$ for details), rather than in the simple error-minimization direction.

It is often used in conjunction with the ER algorithm, alternating several HIO and one or more ER iterations $(\mathrm{HIO}(20)+\mathrm{ER}(1)$ in our case). In particular one or more ER steps are used at the end of the iteration. Elser 43] pointed out that the iterate $\rho^{n}$ can converge to a fixed point $\left(\rho^{n+1}=\rho^{n}\right)$, which may differ from the solution $\bar{\rho}$ $\left(\boldsymbol{P}_{s} \bar{\rho}=\boldsymbol{P}_{m} \bar{\rho}=\bar{\rho}\right)$. However the solution $\bar{\rho}$ can be easily obtained from the fixed point:

$$
\begin{aligned}
\bar{\rho}_{m}^{n} & =\boldsymbol{P}_{m} \rho^{n}, \\
\bar{\rho}_{s}^{n} & =\left(1+\frac{1}{\beta}\right) \boldsymbol{P}_{s} \boldsymbol{P}_{m} \rho^{n}-\frac{1}{\beta} \boldsymbol{P}_{s} \rho^{n},
\end{aligned}
$$

where $\bar{\rho}_{m}$ and $\bar{\rho}_{s}$ should coincide, or else their difference can be used as an error metric. See [43] for further details.

The difference map (DM) is a general set of algorithms [43], which requires 4 projections (two time-consuming modulus constraint projections) (Fig. $3(\mathrm{~d})$ ):

$$
\begin{aligned}
& \rho^{(n+1)}=\left\{\boldsymbol{I}+\beta \boldsymbol{P}_{\boldsymbol{s}}\left[\left(1+\gamma_{s}\right) \boldsymbol{P}_{\boldsymbol{m}}-\gamma_{s} \boldsymbol{I}\right]\right. \\
& \left.-\beta \boldsymbol{P}_{\boldsymbol{m}}\left[\left(1+\gamma_{m}\right) \boldsymbol{P}_{\boldsymbol{s}}-\gamma_{m} \boldsymbol{I}\right]\right\} \rho^{(n)} \text {; }
\end{aligned}
$$

the solution corresponding to the fixed point is described in the same article [43]. We will use in the upcoming tests what Elser suggested as the optimum, with $\gamma_{s}=-\beta^{-1}$ and $\gamma_{m}=\beta^{-1}$.

The averaged successive reflections (ASR) [44] algorithm is:

$$
\rho^{(n+1)}=\frac{1}{2}\left[\boldsymbol{R}_{\boldsymbol{s}} \boldsymbol{R}_{\boldsymbol{m}}+\boldsymbol{I}\right] \rho^{(n)} .
$$

The Hybrid Projection Reflection (HPR) [45] algorithm is derived from a relaxation of the ASR:

$$
\begin{aligned}
\rho^{(n+1)} & =\frac{1}{2}\left[\boldsymbol{R}_{\boldsymbol{s}}\left(\boldsymbol{R}_{\boldsymbol{m}}+(\beta-1) \boldsymbol{P}_{\boldsymbol{m}}\right)\right. \\
& \left.+\boldsymbol{I}+(1-\beta) \boldsymbol{P}_{\boldsymbol{m}}\right] \rho^{(n)}
\end{aligned}
$$

It is equivalent to $\mathrm{HIO}$ if positivity (Section III) is not enforced, but it is written in a recursive form, instead of a case-by-case form such as Eq. (19). It is also equivalent to the DM algorithm for $\gamma_{s}=-1, \gamma_{m}=\beta^{-1}$. Finally the relaxed averaged alternating reflectors (RAAR) algorithm [46]:

$$
\rho^{(n+1)}=\left[\frac{1}{2} \beta\left(\boldsymbol{R}_{\boldsymbol{s}} \boldsymbol{R}_{\boldsymbol{m}}+\boldsymbol{I}\right)+(1-\beta) \boldsymbol{P}_{\boldsymbol{m}}\right] \rho^{(n)} .
$$

For $\beta=1$, HIO, HPR, ASR and RAAR coincide.

The first test is performed on the simplest possible case: find the intersection between two lines. Figure 4 shows the behavior of the various algorithms. The two sets are represented by a horizontal blue line (support) and a tilted black line (modulus). ER simply 


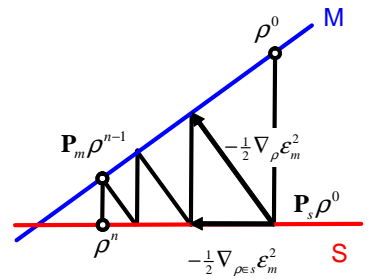

(a)ER

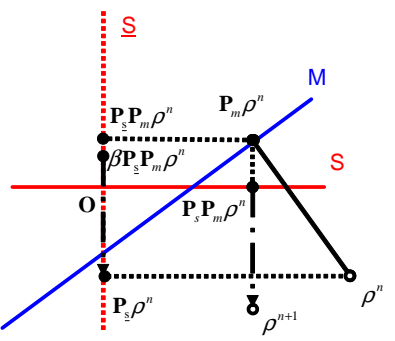

(c) HIO

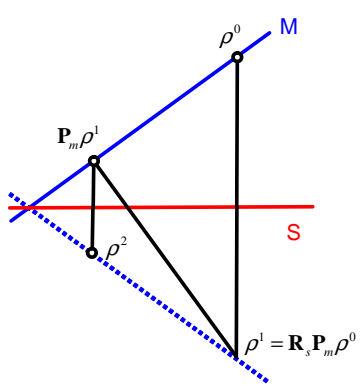

(b) Solvent Flip

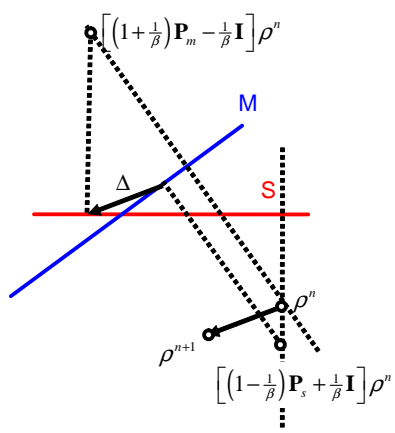

(d)Difference Map
FIG. 3: Geometric representation of various algorithms using a simplified version of the constraint: two lines intersecting. (a) Error reduction algorithm: we start from a point on the modulus constraint by assigning a random phase to the diffraction pattern. The projection onto the modulus constraint finds the point on the set which is nearest to the current one. The arrows indicate the gradients of the error metric. (b) The speed of convergence is increased by replacing the projector on the support with the reflector. The algorithm jumps between the modulus constraint (solid diagonal line) and its mirror image with respect to the support constraint (dotted line). (c) Hybrid input-output, see text (Eq. (19)). The space perpendicular to the support set is represented by the vertical dotted line $\underline{S}$. (d) Difference map, see text (Eq. (22)).

projects back and forth between these two lines, and moves along the support line in the direction of the intersection. SF projects onto the modulus, 'reflects' on the support, and moves along the reflection of the modulus constraint onto the support. The solvent flipping algorithm is slightly faster than ER thanks to the increased in the angle between projections and reflections. HIO and variants (ASR, DM, HPR) move in a spiral around the intersection, eventually reaching the intersection. For similar $\beta$ RAAR behaves somewhere in between ER and HIO with a sharper spiral, reaching the solution much earlier. Alternating 20 iterations of HIO and 1 of $\mathrm{ER}(\mathrm{HIO}(20)+\mathrm{ER}(1))$ considerably speeds up the convergence.

When a gap is introduced between the two lines (Fig. 4(b) , so that they do not intersect, HIO and variants move away from this local minimum in search of another 'attractor' or local minimum. This shows how these algo- rithms escape from local minima and explore the multidimensional space for other minima. ER, SF and RAAR converge to or near the local minimum. By varying $\beta$ RAAR becomes a local minimizer for small $\beta$, and becomes like $\mathrm{HIO}$ for $\beta \simeq 1$. ER, SF and $\mathrm{HIO}+\mathrm{ER}$ converge to the local minimum in these tests.

A more realistic example is shown in Fig. 5. Here the circumference of two circles represents a non-convex set (modulus constraint), while the support constraint is represented by a line. The convex set represents a simplified modulus constraint in a phase-retrieval problem. The advantage of this example is the simplicity in the 'modulus' projector operator (it projects onto the closest circle).

We start from a position near the local minimum. ER, $\mathrm{SF}$, and $\mathrm{HIO}+\mathrm{ER}$ fall into this trap (Fig. 5). HIO and variants move away from the local minimum, 'find' the other circle, and converge to the center of the circle. In the center the projection on the modulus constraint becomes 'multivalued', and its distance metric is 'nonsmooth'. Such a point is unstable, and the algorithms start spiralling toward the solution. For $\beta=0.75$, RAAR does not reach the solution, but converge close to the local minimum.

\section{Positivity}

The situation changes slightly when we consider the positivity constraint. The previous definitions of the algorithms still apply, just replacing $\boldsymbol{P}_{s}$ with $\boldsymbol{P}_{s+}$ :

$$
\boldsymbol{P}_{s+\rho} \rho \boldsymbol{r}= \begin{cases}\rho(\boldsymbol{r}) & \text { if } \boldsymbol{r} \in S \& \rho(\boldsymbol{r}) \geq 0 \\ 0 & \text { otherwise. }\end{cases}
$$

The only difference is for HIO which becomes:

$$
\rho^{(n+1)}= \begin{cases}\boldsymbol{P}_{\boldsymbol{m}} \rho^{(n)}(\boldsymbol{r}) & \text { if } \boldsymbol{r} \in S \& \boldsymbol{P}_{\boldsymbol{m}} \rho^{(n)}(\boldsymbol{r}) \geq 0, \\ \left(1-\beta \boldsymbol{P}_{\boldsymbol{m}}\right) \rho^{(n)} & \text { otherwise. }\end{cases}
$$

$\mathrm{HIO}$ and variants follow the saddle-point direction, moving away from local minima (Fig. 6), but as they approach the solution they react differently to the positivity constraint, with HIO "bounching" at the $x=0$ axis, and ASR/HPR/DM proceeding more smoothly toward the solution.

\section{STEEPEST DESCENT, CONJUGATE GRADIENT, AND MIN-MAX ALGORITHMS}

As discussed in Section III the error reduction (ER) algorithm moves in the direction of the steepest descent [20]; however the step length is not optimized to reach the local minimum in that direction, since it is only one component of the full gradient (Fig. 3(a). Such strategy is generally referred to as reduced gradient method. Figure [7] shows the error metric $\varepsilon_{m}$ as a function of two 


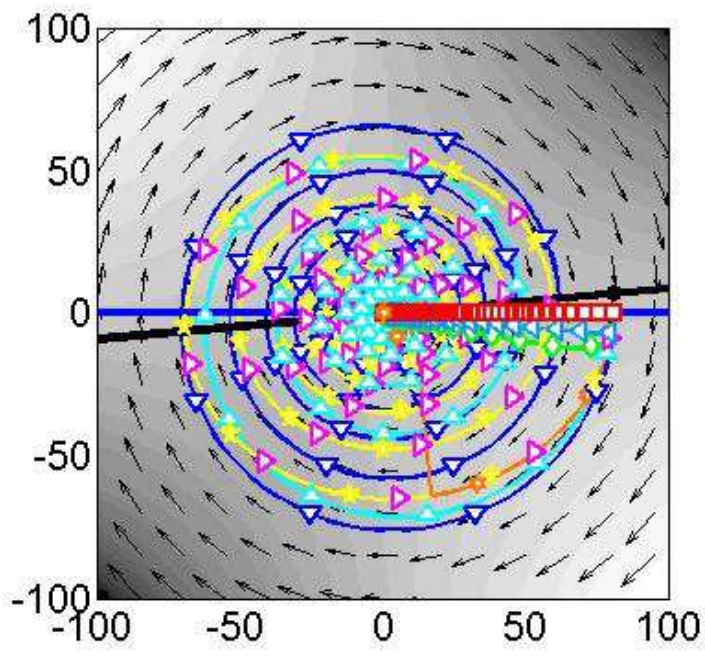

(a)

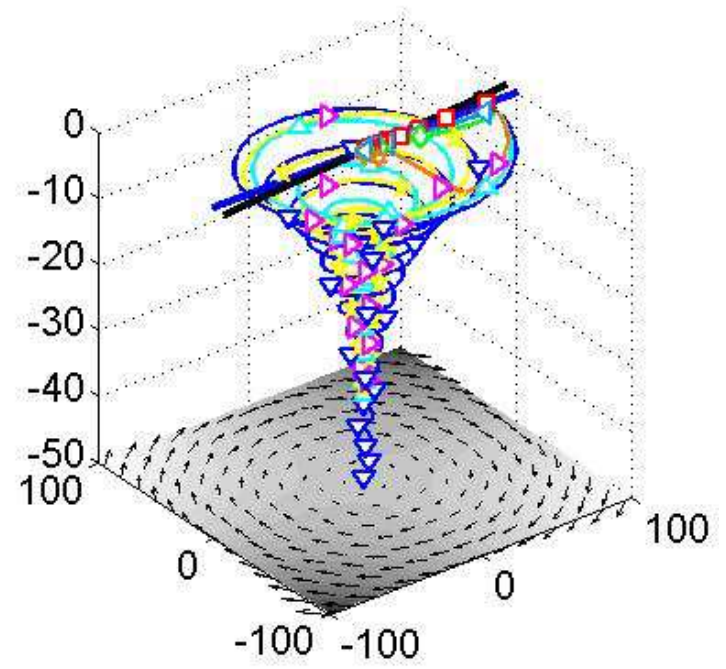

(b)

FIG. 4: The basic features of the iterative projection algorithms can be understood by this simple model of two lines intersecting (a). The aim is to find the intersection. The ER algorithm and the solvent flipping algorithms converge in some gradient-type fashion (the distance to the two sets never increases), the solvent flip method being slightly faster when the angle between the two lines is small. HIO and variants move following a spiral path. The lagrangian $\left(\mathcal{L}=\varepsilon_{m}^{2}-\varepsilon_{s}^{2}\right)$ is represented in grayscale, and the descent-ascent directions $\left(\left[-\nabla_{s}, \nabla_{\underline{s}}\right] \mathcal{L}\right)$ are indicated by arrows. When the two lines do not intersect (b), HIO and variants keep moving in the direction of the gap between the two lines, away from the local minimum. ER, SF and RAAR converge at (or close to) the local minimum.

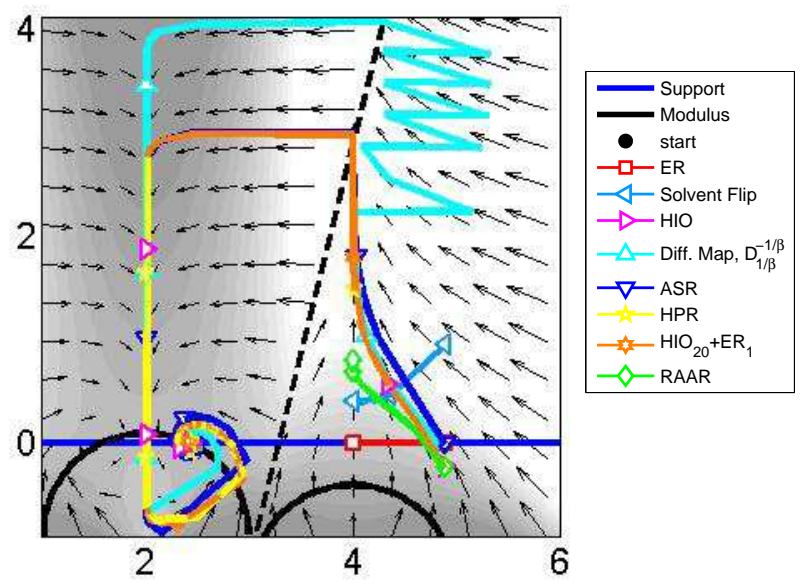

FIG. 5: The horizontal line represents a support constraint, while the two circles represent a non-convex constraint, i.e. the modulus constraint. The gradient-type (ER and SF) algorithms converge to the local minimum, while HIO and variants follow the descent-ascent direction $\left(\left[-\nabla_{s}, \nabla_{\underline{s}}\right] \mathcal{L}\right)$ indicated by the arrows.

unknown pixel values in a simple two-dimensional phaseretrieval problem, and the behavior of the ER algorithm toward the local minima.

The simplest acceleration strategy, the steepest descent method, uses the steepest direction (gradient) and performs a line search of the local minimum in the de-

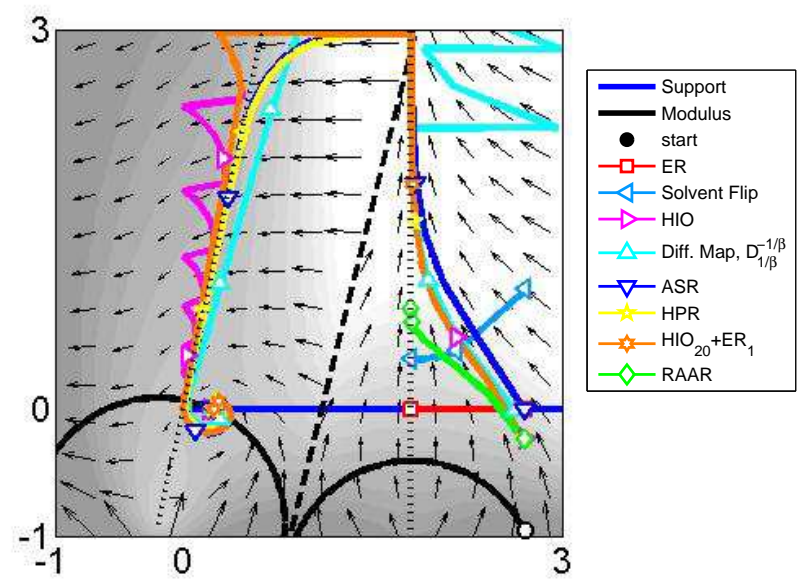

FIG. 6: Positivity constraint: the support constraint is represented by a horizontal line originating from $0(x \geq 0)$. A barrier due to the positivity constraint changes the behavior of the algorithms, which no longer follow the descent-ascent direction. HIO bounces on the $x=0$ axis, while the other algorithms are smoother.

scent direction:

$$
\begin{gathered}
\min _{\delta} \varepsilon_{m}^{2}(\rho+\delta \Delta \rho), \\
\Delta \rho=-\frac{1}{2} \nabla_{s} \varepsilon_{m}^{2}(\rho)=-\boldsymbol{P}_{s}\left[\boldsymbol{I}-\boldsymbol{P}_{m}\right] \rho,
\end{gathered}
$$

where $\nabla_{s}=\boldsymbol{P}_{s} \nabla_{\rho}$ is the gradient with respect to $\rho_{s}$. At a minimum any further movement in the direction of the current step increases the error metric; the gradient 
direction must be perpendicular to the current step. In other words the current step and the next step become orthogonal:

$$
\begin{aligned}
\frac{\partial}{\partial \delta} \varepsilon_{m}^{2}(\rho+\delta \Delta \rho) & =\left\langle\Delta \rho \mid \boldsymbol{P}_{s}\left[\boldsymbol{I}-\boldsymbol{P}_{m}\right]\left(\rho+\delta \Delta \rho_{s}\right)\right\rangle_{r} \\
0 & =\left\langle\Delta \rho_{s} \mid\left[\boldsymbol{I}-\boldsymbol{P}_{m}\right]\left(\rho+\delta \Delta \rho_{s}\right)\right\rangle_{r}
\end{aligned}
$$

where $\langle\boldsymbol{x} \mid \boldsymbol{y}\rangle_{r}=\Re\left(\boldsymbol{x}^{\dagger} \cdot \boldsymbol{y}\right)$. The line search algorithm can use $\epsilon_{m}^{2}$, and/or its derivative in Eq. (29). This optimization should be performed in reciprocal space, where the modulus projector is a diagonal operator and is fast to compute (Eq. (7)), while the support projection requires two Fourier transforms:

$$
\tilde{\boldsymbol{P}}_{s}=\mathcal{F} \boldsymbol{P}_{s} \mathcal{F}^{-1}
$$

The steepest descent method is known to be inefficient in the presence of long narrow valleys, where imposing that successive steps be perpendicular causes the algorithm to zig-zag down the valley. This problem is solved by the non-linear conjugate gradient method [57, 58, 59, 60, 61, 62, 63]. Instead of moving in the direction of steepest descent $\Delta \rho_{s}$, we move in the conjugate direction $\Lambda \rho_{s}$ :

$$
\Lambda \rho_{s}^{(n)}=\left\{\begin{array}{lr}
\Delta \rho_{s}^{(n)} & \text { if } n=1 \\
\Delta \rho_{s}^{(n)}+\gamma_{s} \Lambda \rho_{s}^{(n-1)} & \text { otherwise }
\end{array}\right.
$$

with $\gamma_{s}$ given by the Polak-Ribière method [61]:

$$
\gamma_{s}=\frac{\left\langle\Delta \rho_{s}^{(n)} \mid \Delta \rho_{s}^{(n)}-\Delta \rho_{s}^{(n-1)}\right\rangle_{r}}{\left\|\Delta \rho_{s}^{(n-1)}\right\|^{2}}
$$

and forced to be positive: $\gamma=\max \left(\gamma_{s}, 0\right)$ to improve its reliability. The presence of local minima shown in the previous chapters, however, will cause stagnation of steepest and conjugate gradient methods, preventing global convergence (Fig. 7(c) .

\section{Feedback and the saddle-point problem}

The ability to escape local minima demonstrated by input-output feedback-based algorithms (Fig. 7(d)) makes them superior to the methods based on simple gradient minimization of the error. However, as in the ER algorithm, the step length is not optimized, the algorithm keeps moving in the same direction for several steps, and sometimes overshoots. Combining the ideas of the conjugate gradient or the steepest descent methods and IO feedback could considerably speed-up convergence. Given the lagrangian $\mathcal{L}$ defined as the difference between the two errors:

$$
\mathcal{L}(\rho)=\varepsilon_{m}^{2}(\rho)-\varepsilon_{s}^{2}(\rho),
$$

using equations (13) and (15) we obtain the gradient:

$$
\nabla \mathcal{L}(\rho)=2\left[\boldsymbol{P}_{s}-\boldsymbol{P}_{m}\right] \rho .
$$

The step $\Delta \rho$ used in HIO (Eq. 19) can be expressed in terms of this gradient $\nabla \mathcal{L}$ :

$$
\begin{aligned}
\Delta \rho & =\rho^{(n+1)}-\rho^{(n)} \\
& =\left\{\boldsymbol{P}_{s}\left[\boldsymbol{P}_{m}-\boldsymbol{I}\right]-\beta \boldsymbol{P}_{\underline{s}} \boldsymbol{P}_{m}\right\} \rho, \\
& =\left\{\boldsymbol{P}_{s}\left[\boldsymbol{P}_{m}-\boldsymbol{P}_{s}\right]-\beta \boldsymbol{P}_{\underline{s}}\left[\boldsymbol{P}_{m}-\boldsymbol{P}_{s}\right]\right\} \rho, \\
& =\left\{-\boldsymbol{P}_{s}+\beta \boldsymbol{P}_{\underline{s}}\right\} \frac{1}{2} \nabla \mathcal{L}(\rho) .
\end{aligned}
$$

$\mathrm{HIO} / \mathrm{HPR} / \mathrm{ASR}$ algorithms move toward the minimum of $\mathcal{L}$ in the subspace $\rho_{s}$, and the maximum in the subspace $\rho_{\underline{s}}$, using a reduced gradient optimization strategy, where the step is proportional to the gradient but with one sign reversal (Eq. (35)). In other words, they seek the saddle point:

$$
\min _{\rho_{s}} \max _{\rho_{\underline{s}}} \mathcal{L}\left(\rho_{s}+\rho_{\underline{s}}\right)
$$

Min-max or saddle-point problems arise in fields as various as game theory, economics, physics, engineering, and primal-dual optimization methods. Function minimization is easier than saddle-point optimization because a simple function evaluation can tell us if a new point is better than the previous one. The saddle can be higher or lower than the current value, although the direction toward the saddle is indicated by the two gradient components. One option is to alternate minimization in the direction $\rho_{s}$ and maximization in the direction $\rho_{\underline{s}}$ of $\mathcal{L}$. Such a strategy is similar to alternating HIO and ER algorithms and can be performed using off-the-shelf optimization routines, but it can be slow. Optimization of the step length, a multiplicative factor $\delta$, is obtained by increasing $\alpha$ until the current and next search directions become perpendicular to one another (Fig. 7(e)]:

$$
\begin{aligned}
\left\langle\Delta \rho \mid\left[\boldsymbol{P}_{s}-\beta \boldsymbol{P}_{\underline{s}}\right] \nabla \mathcal{L}(\rho+\delta \Delta \rho)\right\rangle_{r} & =0, \\
\left\langle\Delta \rho \mid\left\{\boldsymbol{P}_{s}\left[\boldsymbol{P}_{m}-\boldsymbol{I}\right]-\beta \boldsymbol{P}_{\underline{s}} \boldsymbol{P}_{m}\right\}(\rho+\delta \Delta \rho)\right\rangle_{r} & =0 .
\end{aligned}
$$

In analogy to the conjugate gradient method, one could substitute the search direction $\Delta \rho$ with $\Lambda \rho$, as in Eq. (31) (Fig. 7(f) . A more robust strategy involves replacing the one-dimensional search with a two-dimensional optimization of the saddle point (Fig. 7(g)):

$$
\begin{array}{r}
\min _{\alpha} \max _{\beta} \psi(\alpha, \beta), \\
\psi(\alpha, \beta)=\mathcal{L}\left(\rho+\alpha \Delta \rho_{s}+\beta \Delta \rho_{\underline{s}}\right) .
\end{array}
$$

Once the 2D min-max problem is solved, the new directions can be obtained by following the conjugate gradient scheme (Fig. 7(i).

\section{CONCLUSIONS}

Lensless imaging owes its success as an effective tool to observe nanoscale systems to the advances made in phase-retrieval algorithms. The new instruments replacing lenses are the iterative projection algorithms for 


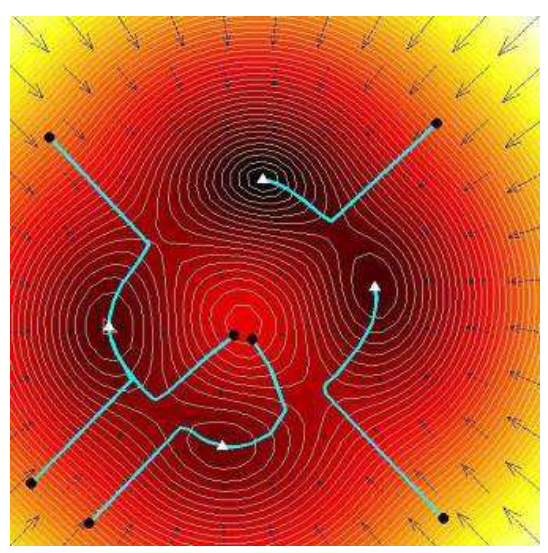

(a)Reduced Gradient Method (ER)

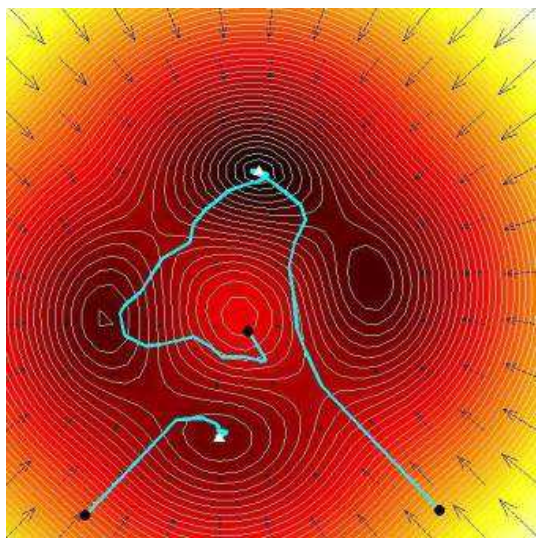

(d)Reduced gradient saddle optimization (HIO/ASR)

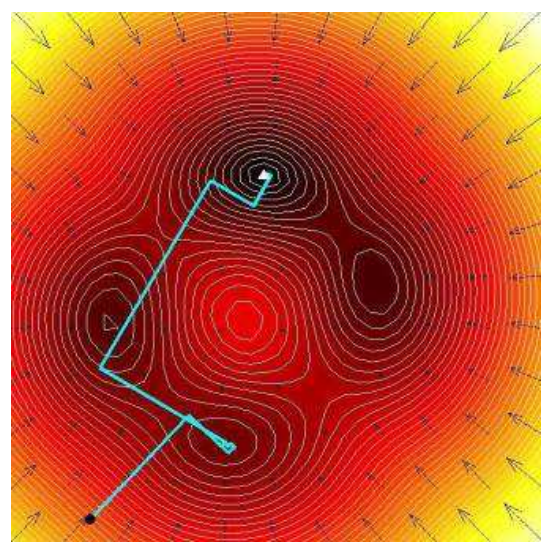

(g)2D saddle optimization

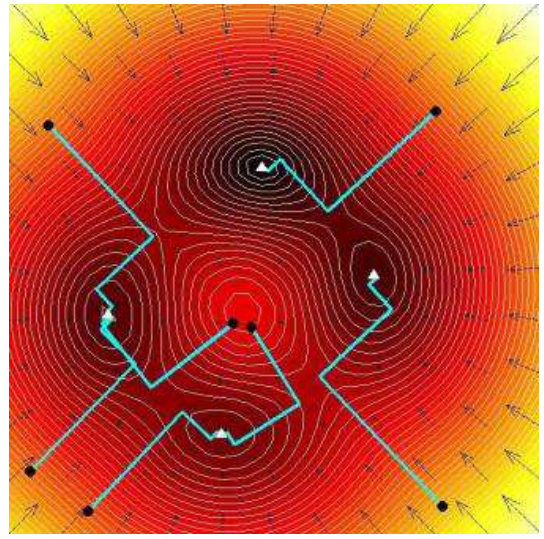

(b)Steepest Descent

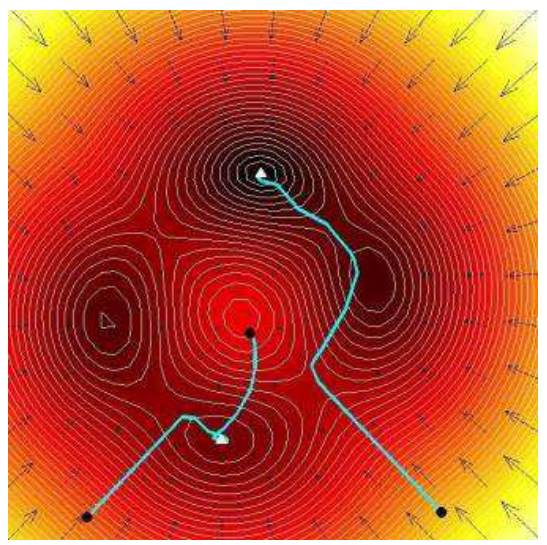

(e)1D saddle optimization

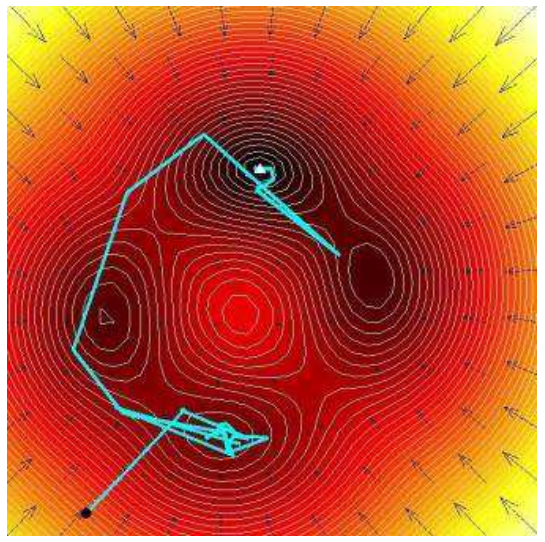

(h)2D conjugate saddle optimization (1)

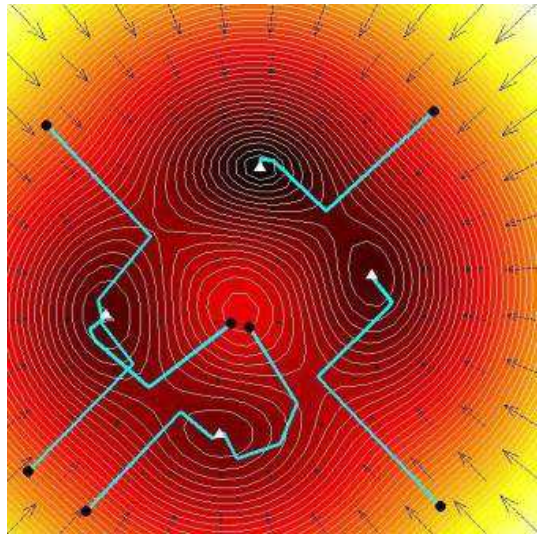

(c)Conjugate Gradient

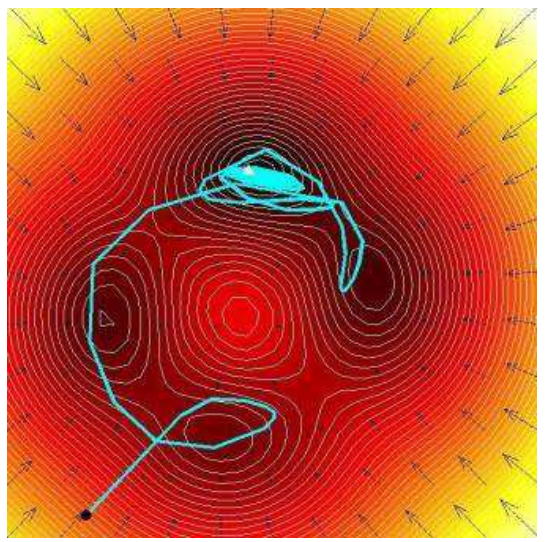

(f)Conjugate saddle optimization

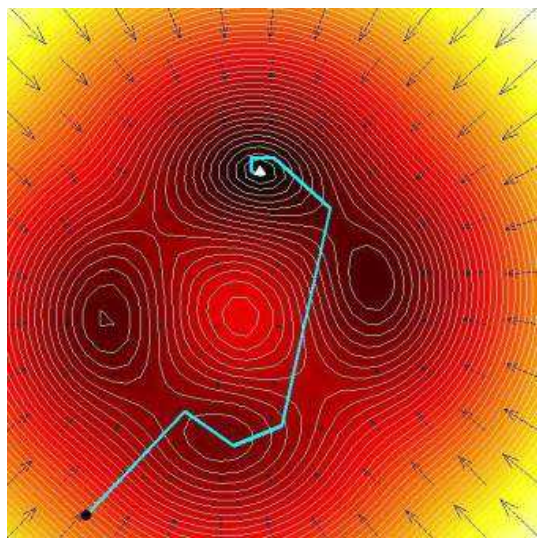

(i)2D conjugate saddle optimization (2)

FIG. 7: A simple 2-D phase-retrieval problem: only two variables (pixel values) are unknown. The solution - the global minimum - is the top minimum in the figures. The colormap and contour lines represents the error metric $\varepsilon_{m}\left(\rho_{s}\right)$, and the descent direction is indicated by the arrows. The error reduction algorithm (a) proceeds toward the local minimum without optimizing the step length and stagnates at the local minima. The steepest descent method (b) moves toward the local minimum with a zig-zag trajectory, while the conjugate gradient method reaches the solution faster (c). The HIO method generally converges to the global minimum, however some rare starting points converge to a local minimum (d). The saddlepoint optimization with optimized step length (Eq. 37) stagnates in the same local minimum as HIO (e). The conjugate gradient version avoids stagnation (f). The saddle point optimization using a two dimensional search of the saddle point reaches the global minimum from a larger range of starting points than HIO (g). The conjugate gradient version (h, i) reaches the solution faster if the conjugate directions $\Lambda \tilde{\rho}_{s, \underline{s}}$ are obtained independently from $\Delta \tilde{\rho}_{s, \underline{s}}$ (i), rather than their sum $\Delta \tilde{\rho}=\Delta \tilde{\rho}_{s}+\Delta \tilde{\rho}_{\underline{s}}$. 


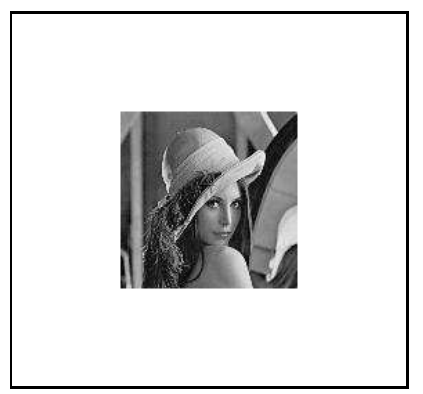

FIG. 8: Test figure used for benchmarking. The object of $128^{2}$ elements is surrounded by empty space. The whole image has $256^{2}$ elements. The Fourier transform of this image provides the data set, and its area defines the support

phase retrieval. These algorithms can be grouped in two categories: (1) local minimizers such as ER, SF, steepest descent and conjugate-gradient methods, with Solvent Flip having some moderate ability to escape local minima [55]. (2) more global minimizers such as HIO, DM, ASR, HPR which use a feedback to reach the solution. RAAR and ER+HIO fall somewhere in between the two categories, depending on an adjustable parameter. A simple benchmark is shown for comparison in Fig. 8 and summarized in Table II. The test consisted in solving a phase-retrieval problem without assuming positivity (nor reality) of the object, and the support region was slightly larger than the object, and was repeated 100 times for each algorithm. Many algorithms surprisingly failed, and only the ones shown in Fig. 9]succeeded. HIO appears to be the most effective algorithm, and it is significantly improved in terms of speed and reliability when the two-dimensional step size optimization (SO2D), as described in Eq. (38) is applied. Further improvements in reliability are achieved by performing a saddle-point optimization in a 4-dimensional space of two successive steps (SO4D). Minimization algorithms, although not very powerful at solving the phase problem, can be used to polish-up a solution, improving the values of the error metric considerably. The algorithms described here use as prior knowledge the support region. Algorithms that use a simple threshold to replace the support [32, 56] or more sophisticated support refinement [34] have not been discussed. Various projection algorithms combined with some form of threshold have produced remarkable reconstructions of single isolated objects (HIO and RAAR [30], DM [27]), as well as single and powder crystals (SF and HIO with support refinement [56, 64, 65]), but a full comparison of the algorithms behavior applied to this type of constraint have not been discussed.

\section{Acknowledgments}

This work was performed under the auspices of the U.S. Department of Energy by the Lawrence Livermore

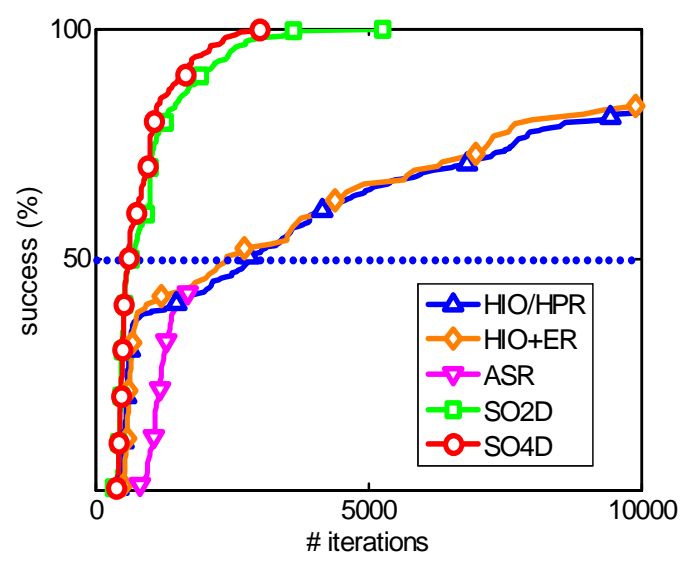

FIG. 9: Percentage of successful reconstructions over many tests starting from random phases as a function of number of iterations. The support is the only constraint. Positivity and reality are not enforced, and the support is loose: it is larger than the object by one additional row and column

TABLE II: Benchmark of various algorithms

\begin{tabular}{|l|l|l|}
\hline Algorithm & $\begin{array}{l}\text { No. of iterations for } \\
50 \% \text { success }\end{array}$ & $\begin{array}{l}\text { success after } \\
10000 \text { iterations }\end{array}$ \\
\hline HIO/HPR & 2790 & $82 \%$ \\
HIO/HPR+ER & 2379 & $82.6 \%$ \\
ASR & $1697^{a}$ & $42 \%$ \\
SO2D & 656 & $100 \%$ \\
SO4D & 605 & $100 \%$ \\
Others & $>10000$ & $0 \%$ \\
\hline
\end{tabular}

${ }^{a} 42 \%$ success, the algorithm either reconstruct in a limited number of iterations or never

National Laboratory under Contract No. W-7405-ENG48 and the Director, Office of Energy Research. This work was partially funded by the National Science Foundation through the Center for Biophotonics. The Center for Biophotonics, a National Science Foundation Science and Technology Center, is managed by the University of California, Davis, under Cooperative Agreement No. PHY0120999. The author acknowledge useful discussions with H. N. Chapman, M. R. Howells, J. C. H. Spence and D. R. Luke.
[1] M. R. Howells, T. Beetz, H. N. Chapman, C. Cui, J. M. Holton, C. J. Jacobsen, J. Kirz, E. Lima, S. Marchesini, H. Miao, D. Sayre, D. A.
Shapiro, J. C. H. Spence, arXiv:physics/0502059 (http://arxiv.org/pdf/physics/0502059).

[2] J. C. Solem and G. C. Baldwin, Science 218, 229 (1982). 
[3] R. Neutze, R. Wouts, D. van der Spoel, E. Weckert \& J. Hajdu, Nature 406, 752-757 (2000).

[4] H. N. Chapman, A Barty, M. J. Bogan, S. Boutet, M. Frank, S. P. Hau-Riege, S. Marchesini, B. W. Woods, S. Bajt, W. H. Benner, R. A. London, E. Plönjes, M. Kuhlmann, R. Treusch, S Düterer, T. Tschentscher, J. R. Schneider, E. Spiller, T. Möller, C. Bostedt, M. Hoener, D. A. Shapiro, K. O. Hodgson, D. Van Der Spoel, F. Burmeister, M. Bergh, C. Caleman, Gösta Huldt, M. M. Seibert, F. R. N. C. Maia, R. W. Lee, A. Szöke, N. Timneanu, Janos Hajdu, Nature Physics 2, 789-862 (2006), arxiv:physics/0610044.

[5] J. C. H. Spence, R. B. Doak, Phys. Rev. Lett. 92, 198102 (2004).

[6] R. Gerchberg and W. Saxton, Optik 35, 237 (1972).

[7] J. R. Fienup, Opt. Lett. 3, 27 (1978).

[8] J. R. Fienup, J. C. Marron, T. J. Schulz and J. H. Seldin, Appl. Opt. 32, 1747-1768 (1993).

[9] H. M. Quiney, A. G. Peele, Z. Cai, D. Paterson and K. A. Nugent, Nature Physics 2, 101 - 104 (2006).

[10] J. C. H. Spence, U. Weierstall , M. Howells , Phil. Trans. A 360, 875-895 (2002).

[11] J. M. Zuo, I. Vartanyants, M. Gao, R. Zhang and L.A. Nagahara, Science 300, 1419-1421 (2003).

[12] J. Wu, U. Weierstall, J. C. H. Spence, Nature Materials 4, 912 (2005).

[13] R. P. Millane, J. Opt. Soc. Am. A 7, 394 (1990).

[14] International Tables for Crystallography Vol. F, Ed. M. G. Rossmann and E. Arnold, The International Union of Crystallography, Kluwer Academic Publishers, Dordrecht/Boston/London, (2001).

[15] R. H. T. Bates, Optik 61, 247 (1982).

[16] D. Sayre, Acta Cryst. 5, 843 (1952).

[17] D. Sayre, "Prospects for long-wavelength x-ray microscopy and diffraction", in Imaging Processes and Coherence in Physics, Eds. M. Schlenker, M. Fink, J. P. Goedgebuer, C. Malgrange, J. C. Viénot, R. H. Wade, Lecture Notes in Physics 112, 229-235, (Springer-Verlag, Berlin, 1980).

[18] J. Miao, D. Sayre and H. N. Chapman, J. Opt. Soc. Am A 15, 1662 (1998).

[19] J. R. Fienup, Opt. Eng. 19, 297 (1980).

[20] J. R. Fienup, Appl. Opt. 21, 2758 (1982).

[21] J. Miao, P. Charalambous, J. Kirz, D. Sayre, Nature 400, 342 (1999).

[22] G. J. Williams, M. A. Pfeifer, I. A. Vartanyants and I. K. Robinson, Phys. Rev. Lett. 90, 175501 (2003).

[23] M. A. Pfeifer, G. J. Williams, I. A. Vartanyants, R. Harder \& I. K. Robinson, Nature 442, 63-67 (2006).

[24] J. Miao, C-C. Chen, C. Song, Y. Nishino, Y. Kohmura, T. Ishikawa, D. Ramunno-Johnson, T-K. Lee, and S. H. Risbud, Phys. Rev. Lett. 97, 215503 (2006).

[25] J. W. Miao, K. O. Hodgson, T. Ishikawa, C. A. Larabell, M. A. LeGros, Y. Nishino, Proc. Nat. Ac. Sci. 100, 110 (2003).

[26] T. Beetz, M. R. Howells, C. Jacobsen, C.-C. Kao, J. Kirz, E. Lima, T. O. Mentes, H. Miao, C. Sanchez-Hanke, D. Sayre, D. Shapiro, Nucl. Instrum. Meth. A 545, 459 (2005).

[27] D. Shapiro, P. Thibault, T. Beetz, V. Elser, M. Howells, C. Jacobsen, J. Kirz, E. Lima, H. Miao, A. Neiman, D. Sayre, PNAS 102 (43), 1543 (2005).

[28] E. Lima, D. Shapiro, P. Thibault, T. Beetz, V. Elser, M. Howells, X. Huang, C. Jacobsen, J. Kirz, H. Miao, A. M.
Neiman, A. Stewart and D. Sayre, IPAP Conf. Series 7, 392-395 (2006).

[29] M. R. Howells, P. Charalambous, H. He, S. Marcesini, J C. H. Spence, Proc. SPIE 4783, 65 (2002).

[30] H. N. Chapman, A. Barty, S. Marchesini, A. Noy, C. Cui, M. R. Howells, R. Rosen, H. He, J. C. H. Spence, U. Weierstall, T. Beetz, C. Jacobsen, D. Shapiro, J. Opt. Soc. Am. A 23, 1179-1200 (2006), arXiv:physics/0509066

[31] A. Barty et al., "Three-dimensional ceramic nanofoam lattice structure determination using coherent X-ray diffraction imaging: insights into deformation mechanisms", submitted.

[32] R. P. Millane, J. Opt. Soc. Am. A 13, 725 (1996).

[33] W. McBride, N. L. O'Leary, and L. J. Allen, Phys. Rev. Lett. 93, 233902 (2004).

[34] S. Marchesini, H. He, H. N. Chapman, S. P. HauRiege, A. Noy, M. R. Howells, U. Weierstall and J.C.H. Spence Phys. Rev. B 68, 140101(R) (2003), arXiv:physics/0306174

[35] J. C. H. Spence, U. Weierstall, and M. R. Howells, Ultramicros. 101, 149 (2004).

[36] J. W. Goodman, "Statistical Optics" New York: Wiley (1985).

[37] R. Barakat and G. Newsam, J. Math. Phys. 25, 31903193 (1984).

[38] M. J. Buerger, Vector space and its application in crystal structure investigation', (Wiley, New York, 1959).

[39] S. Marchesini, H. N. Chapman, A. Barty, M. R. Howells, J. C. H. Spence, C. Cui, U. Weierstall, and A. M. Minor, IPAP Conf. Series 7, 380-382 (2006) arXiv:physics/0510033

[40] J. N. Cederquist, J. R. Fienup, J. C. Marron, R. G. Paxman, Opt. Lett. 13, 619 (1988).

[41] A. Levi and H. Stark, J. Opt. Soc. Am. A 1, 932-943 (1984).

[42] H. Stark, Image Recovery: Theory and applications (Academic Press, New York, 1987).

[43] V. Elser, J. Opt. Soc. Am. A 20, 40 (2003).

[44] H. H. Bauschke, P. L. Combettes, and D. R. Luke. J. Opt. Soc. Am. A 19, 1334-1345 (2002).

[45] H. H. Bauschke, P. L. Combettes and D. R. Luke, J. Opt. Soc. Am. A 20, 1025-1034 (2003).

[46] D. R. Luke, Inverse Problems 21, 37-50(2005), arXiv:math.OC/0405208.

[47] S. P. Hau-Riege, H. Szöke, H. N. Chapman, A. Szöke, S. Marchesini, A. Noy, H. He, M. R. Howells, U. Weierstall, J. C. H. Spence, Acta Cryst. A60, 294-305 (2004), arXiv:physics/0403091

[48] D. R. Luke, J. V. Burke, R. G. Lyon, SIAM Review 44, 169-224 (2002).

[49] J. V. Burke and D. R. Luke. SIAM J. Control Opt. 42, 576-595 (2003).

[50] P.-T. Chen, M. A. Fiddy, C.-W. Liao and D. A. Pommet, J. Opt. Soc. Am. A 13, 1524-31 (1996).

[51] J. R. Fienup, C. C. Wackerman, J. Opt. Soc. Am. A 3, 1897-1907 (1986).

[52] T. Isernia, G. Leone, R. Pierri and F. Soldovieri, J. Opt. Soc. Am. A 16, 1845-1856 (1999).

[53] G. Oszlányi and A. Süto, Acta Cryst. 61, 147-152 (2005).

[54] L. M. Brègman, Sov. Math. Dokl. 6, 688-692 (1965).

[55] J. P. Abrahams and A. W. G. Leslie, Acta Cryst. D52, 30-42 (1996).

[56] G. Oszlányi and A. Süto, Acta Cryst. A60, 134-141 (2004), arXiv:cond-mat/0308129 
[57] W. H. Press, S. A. Teukolsky, W. T. Vetterling and B. P. Flannery, Numerical Recipes in $C$, (Cambridge University Press 1992).

[58] M. R. Hestenes, Conjugate Direction Methods in Optimization, (Springer-Verlag, New York, 1980).

[59] Fletcher, R. and Reeves, C.M., Comp, J. 7, 149-154 (1964).

[60] M. J. D. Powell, Lecture Notes in Mathematics 1066, 122-141 (1984).

[61] E. Polak, G. Ribiére, Revue Française d'Informatique et de Recherche Opérationelle 16, 35 (1969).

[62] R. Fletcher, and C. M. Reeves, Comp, J. 7, 149-154 (1964).

[63] E. Polak, Computational Methods in Optimization (New York: Academic Press 1971).

[64] J. S. Wu, J. C. H. Spence, M. O'Keeffe and T. L. Groy Acta Cryst. A60, 326-330 (2004).

[65] J. Wu, K. Leinenweber, J. C. H. Spence, Nature Materials 5, 647-652 (2006). 